\section{Should the UK introduce a universal childhood varicella vaccination programme?}

\author{
Gayatri Amirthalingam, Mary Ramsay
}

Primary varicella infection (chickenpox) is common in the UK with over threequarters of parents reporting a history of chickenpox in their children by 5 years of age. ${ }^{1}$ Following primary infection, the varicella zoster virus (VZV) remains dormant in the dorsal root ganglia and reactivates in later life following a decline in cell-mediated immunity to cause herpes zoster or shingles (HZ). Although chickenpox is generally mild and selflimiting in healthy children, secondary bacterial infections, pneumonia and neurological complications can occur. The risk of severe chickenpox is higher in immunocompromised individuals, pregnant women and neonates, although most hospitalisations for severe complications are in previously healthy children. ${ }^{2}$ Shingles is a potentially debilitating condition, which results in a greater burden and quality of life loss than chickenpox. ${ }^{3}$ The incidence of shingles and the risk of post herpetic neuralgia increase with age.

Safe and effective live-attenuated varicella vaccines (Oka VZV strain) have been

Immunisation, Hepatitis and Blood Safety Department, Public Health England, London, UK

Correspondence to Dr Gayatri Amirthalingam, Immunisation, Hepatitis and Blood Safety Department, Public Health England, 61 Colindale Avenue, London NW9 5EQ, UK; Gayatri.amirthalingam@phe.gov.uk available for the prevention of chickenpox since the 1980s; two doses have a reported effectiveness between $84 \%$ and 98\%. ${ }^{4}$ Countries across Europe, North America and Australia have adopted different approaches to using vaccine for VZV control. While some countries, such as Australia and the USA, have introduced routine childhood varicella programmes using one or two dose schedules, many European countries (including the UK and Belgium) have not. In the UK, a selective vaccination policy has been recommended, offering vaccine to high-risk groups including non-immune healthcare workers and susceptible household contacts of immunosuppressed individuals. ${ }^{5}$

The USA, which initially introduced a one-dose varicella programme in 1995, saw a significant decline in varicellarelated deaths in 1-4 year olds (92\% fall between 1990-1994 and 1999-2001), hospitalisations (88\% fall between 1994/ 1995 and 2002) and ambulatory visits (59\% fall between 1994/1995 and 2002). ${ }^{4}$ Similar declines have been observed in other countries using a one-dose schedule such as Australia. ${ }^{6} \mathrm{~A}$ potential concern with a one-dose programme where sufficiently high coverage is not achieved, however, is a shift in the average age at infection to older age groups where the disease is likely to be more severe. In the USA where coverage in the early years was suboptimal, the average age at infection increased from 3-6 years in 1995 to 9-11 years in 2005, although the age-specific incidence decreased in all age groups. ${ }^{7}$ Despite the success of the one-dose programme in the USA, breakthrough infections and outbreaks in vaccinated populations were observed and in 2006, given the evidence of higher vaccine effectiveness, a two-dose policy was adopted. Because of an increased risk of febrile seizures observed with combined measles, mumps, rubella and varicella vaccine at $12-15$ months of age, the Centers for Disease Control and Prevention currently recommends that the first dose be offered as a monovalent vaccine with the combined vaccine used for the second dose at 4-6 years of age. In the first five years after the second dose was introduced, varicella incidence reached the lowest since the start of the vaccine programme (declining around a further 70\%), with fewer outbreaks and severe cases. ${ }^{8}$

For a vaccine to be recommended for inclusion into the UK routine schedule, evidence of cost effectiveness is required. Accurate assessment of the burden of disease is essential to inform this cost effectiveness. Blumental and colleagues studied varicella-related hospitalisations in 101 hospitals (representing 97.7\% total paediatric beds in Belgium) over 1 year. ${ }^{9}$ The incidence of paediatric varicella hospitalisations was estimated at 29.5 per 100000 person-years-highest in children aged 0-4 years. This compares with a recent retrospective study in England that found rates of hospitalised varicella of 31.2 per 100000 children aged 0-15 years in 2010/2011. ${ }^{10}$ The majority of 
hospitalisations occurred in otherwise healthy children, a feature that is consistent with countries such as the UK. The most common complications were bacterial skin and soft tissue infections, and neurological complications (including meningitis and encephalitis) and pneumonia. While infection resolved in the overwhelming majority of cases, $9 \%$ of children had significant scarring and rarer sequelae such as ataxia and reactive arthritis were also reported. The low rate of severe disease was also seen in prospective surveillance in the UK where hospitalisation occurred at a rate of 0.82 per 100000 children aged $0-4$ years in 20022003. ${ }^{2}$ The estimated varicella-related mortality based on death registrations in Belgium was 0.5 per 1000000 . The authors acknowledge that, given the secular variation that occurs with varicella, assessment of burden based on a single year is a limitation. Between 2001/ 2002 and 2010/2011, varicella hospitalisations in England varied between 57.5 per 1000000 to 78 per $1000000 .^{10}$ Any economic model should therefore allow for a variation of at least 33\%. In addition, as the majority of varicella cases are not hospitalised, it is important to also use data on primary care consultations.

In 2009, the Joint Committee on Vaccination and Immunisation undertook a review of varicella control strategies including the (i) introduction of a two-dose childhood varicella programme, (ii) introduction of a single-dose $\mathrm{HZ}$ programme for older adults and (iii) a combined childhood varicella and adult $\mathrm{HZ}$ programme. The economic analysis indicated that the programme with two doses of varicella vaccine in childhood would not be cost effective in the short to medium term. This conclusion was largely down to a predicted temporary increase in $\mathrm{HZ}$ in the first 30-50 years as a result of reduced VZV exposure. ${ }^{11}$ However, a routine $\mathrm{HZ}$ vaccination programme for adults aged 70 years, with a catch-up programme for those aged 71-79 years, was recommended and implemented in 2013.

The potential increase in $\mathrm{HZ}$ is based on the premise that re-exposure to VZV protects individuals against shingles. This 'exogenous boosting' hypothesis was first postulated in the 1960s by HopeSimpson. Since then, a number of studies have demonstrated the existence of exogenous boosting, although the magnitude of its effect has been difficult to determine. ${ }^{12}$ Studies assessing age-specific trends in $\mathrm{HZ}$ from a number of countries with and without varicella vaccination programmes have also suggested an increase in $\mathrm{HZ}$ incidence over time. ${ }^{4}$ In contrast, the incidence of $\mathrm{HZ}$ based on primary care consultations has been remarkably consistent for decades in England. ${ }^{13}$ In the USA, some authors have reported that $\mathrm{HZ}$ incidence was actually increasing prior to the introduction of the varicella vaccine programme, and therefore, that the increase cannot be attributed to the vaccine programme. Modelling studies predicted that an increase in $\mathrm{HZ}$ would only be observed after running a vaccine programme with high coverage for at least 15 years. With the longest running universal varicella programme, the USA provides the best opportunity to assess any impact on $\mathrm{HZ}$ incidence. However, coverage was $<80 \%$ for the first five years of the US programme, and any predicted increase would only be expected from around now. Further investigation is therefore required to fully understand these observed increases, including an assessment of any changes in ascertainment and in the demographics and risk factor breakdown over time. As the bulk of the predicted increases will be in adults not currently eligible for $\mathrm{HZ}$ vaccination, it is critical to adequately address this serious concern, prior to any decision on the best way to use varicella vaccination in the UK.

Contributors GA reviewed the published literature and undertook the initial draft of the manuscript. Both authors made critical revisions to the manuscript and MR made the final approval prior to submission.

Provenance and peer review Commissioned; internally peer reviewed.

To cite Amirthalingam G, Ramsay M. Arch Dis Child 2016;101:2-3.

Received 3 July 2015

Revised 10 August 2015

Accepted 13 August 2015

Published Online First 4 September 2015

\section{Linked}

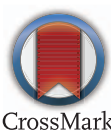

http://dx.doi.org/10.1136/archdischild-2015-308283

Arch Dis Child 2016;101:2-3.

doi:10.1136/archdischild-2015-308730

\section{REFERENCES}

1 Manikkavasagan G, Dezateux C, Wade A, et al. The epidemiology of chickenpox in UK 5-year olds: an analysis to inform vaccine policy. Vaccine 2010;28:7699-705.

2 Cameron JC, Allan G, Johnston F, et al. Severe complications of chickenpox in hospitalised children in the UK and Ireland. Arch Dis Child 2007:92:1062-6.

3 Bilcke J, Ogunjimi B, Marais C, et al. The health and economic burden of chickenpox and herpes zoster in Belgium. Epidemiol Infect 2012;140:2096-109.

4 SAGE Working Group on Varicella and Herpes Zoster Vaccines: Background Paper on Varicella Vaccine SAGE review 2015. http://www.who.int/ immunization/sage/meetings/2014/april/1_SAGE varicella_background_paper_FINAL.pdf (accessed 2/ 07/2015).

5 Public Health England. Shingles. Chapter 28a. Immunisation against Infectious Disease. https:// www.gov.uk/government/uploads/system/uploads/ attachment_data/file/357155/Green_Book_Chapter_ 28a_v0_5.pdf (accessed 2/07/2015).

6 Heywood AE, Wang H, Macartney KK, et al. Varicella and herpes zoster hospitalizations before and after implementation of one-dose varicella vaccination in Australia: an ecological study. Bull World Health Organ 2014;92:593-604.

7 Guris D, Jumaan AO, Mascola L, et al. Changing varicella epidemiology in active surveillance sitesUnited States, 1995-2005. J Infect Dis 2008;197 (Suppl 2):S71-5.

8 Bialek SR, Perella D, Zhang J, et al. Impact of a routine two-dose varicella vaccination program on varicella epidemiology. Pediatrics 2013;132: e1134-40.

9 Blumental S, Sabbe M, Lepage P, the Belgium Group for Varicella. Varicella paediatric hospitalisations in Belgium: a 1-year national survey. Arch Dis Child 2016;101:16-22.

10 Abdalrahman B, Laverty AA, Beckett G, et al. Trends in hospital admissions for Varicella and Zoster viruses in England, 2001/2002-2010/2011: time trend study. JRSM Open 2015;6:2054270414562984.

11 van Hoek AJ, Melegaro A, Gay N, et al. The cost-effectiveness of varicella and combined varicella and herpes zoster vaccination programmes in the United Kingdom. Vaccine 2012;30:1225-34.

12 Ogunjimi B, Van Damme P, Beutels P. Herpes zoster risk reduction through exposure to chickenpox patients: a systematic multidisciplinary review. PLOS ONE 2013;8:e66485.

13 Brisson M, Edmunds WJ. Epidemiology of Varicella-Zoster Virus in England and Wales. J Med Virol 2003;70(Suppl 1):S9-14. 